\title{
Ricci Flows and Solitonic pp-Waves
}

\author{
Sergiu I. Vacaru* \\ Department of Mathematics, Brock University, \\ St. Catharines, Ontario, Canada L2S 3A1 \\ and \\ The Fields Institute for Research in Mathematical Science, \\ 222 College Street, Second Floor, \\ Toronto, Ontario, Canada M5T 3J1
}

September 20, 2006

\begin{abstract}
We find exact solutions describing Ricci flows of four dimensional pp-waves nonlinearly deformed by two/three dimensional solitons. Such solutions are parametrized by five dimensional metrics with generic off-diagonal terms and connections with nontrivial torsion which can be related, for instance, to antisymmetric tensor sources in string gravity. There are defined nontrivial limits to four dimensional configurations and the Einstein gravity.
\end{abstract}

Keywords: Ricci flows; gravitational solitons; pp-waves.

PACS numbers: 04.20.Jb, 04.30.Nk, 04.50.+h, 04.90.+e, 02.30.Jk

\section{Introduction}

Solutions with pp-waves [1] and their generalizations are of special interest in modern gravity and string theory in curved backgrounds [2]. In this paper, we consider three classes of five dimensional (5D) metrics describing Ricci flows of solitonic pp-waves (see Refs. [3] and [4] for reviews of results on

*Permanent: 108-1490 Eginton Ave., Toronto, M6E 2G5, Canada

e-mails: sergiu_vacaru@yahoo.com, svacaru@brocku.ca and svacaru@fields.utoronto.ca 
gravitational solitons and, respectively, Ricci flows). For non-solitonic configurations, a subclass of such solutions has limits to the trivial embedding of the $4 \mathrm{D}$ pp-wave metrics into 5D spacetimes.

There were elaborated various methods of constructing exact solutions in gravity, see a summary of main approaches in Refs. [5] and [6]. The bulk of such solutions are with a prescribed symmetry (spherical, cylindrical, torus, Killing or a Lie algebra ones ...) and generated by diagonalizable (by coordinated transforms) ansatz for metrics. Such ansatz reduce the Einstein equations to certain classes of nonlinear ordinary differential equations and/or algebraic equations which can be solved exactly. Then, an interesting discovery was the existence of an infinite-dimensional group of transformations of metrics mapping a solution of the Einstein equations to another solution (Geroch group [7]).

In our works [8], we developed a general geometric method of constructing exact solutions by using anholonomic deformations of metrics (they may define, or not, any exact solutions) to certain classes of generic off-diagonal metrics exactly solving the gravitational field equations in a model of gravity theory (we can consider the Einstein general relativity or various type of string, gauge,... both commutative and noncommutative generalizations). Such spacetimes, in general, do not possess any Killing/ group symmetry but can be characterized by generalized symmetries induced by 'anholonomy' relations for preferred frames with associated nonlinear connection structure. ${ }^{1}$ The exact off-diagonal solutions (for instance, in 4D and/or 5D) constructed following the 'anholonomic frame method' depend on classes of functions on 2-4 variables and can be restricted to possess noncommutative symmetries, generalized Lie symmetries like those for the Lie, or Clifford, algebroids. The papers [8, 9, 10] contain details and references on recent developments on constructing black ellipsoid and black torus solutions, locally anisotropic wormholes, nonholonomically deformed Taub NUT spacetimes and so on.

The aim of this paper is to show how we can apply the anholonomic frame method in order to generate 5D exact solutions of the Ricci flows equations describing nonlinear, for instance, solitonic deformations of $4 \mathrm{D}$ pp--waves ${ }^{2}$ as flows in 5D gravity. We shall define the conditions when such solutions possess nontrivial limits to the Einstein gravity or their torsion can be related

\footnotetext{
${ }^{1}$ The geometric constructions on spaces provided with anholonomic structure can be performed with resect to any frames of reference and for any local systems of coordinates. The term 'preferred' emphasizes that there are certain classes of 'natural' frames and coordinates when a symmetry of fixed type can be distinguished in explicit form and the field equations can be exactly integrated.

${ }^{2}$ in a similar form we can consider generalizations to Kaigorodov spacetimes 11] and certain higher dimension solutions [2]
} 
to the antisymmetric 'H-field' in string gravity.

The paper is arranged as follows. After outlining some results on Ricci flows for generic off-diagonal metrics in section 2 , we consider $4 \mathrm{D}$ pp-wave metrics and analyze their flows on a time like coordinate $v=z-t$ in 5D spaces with possible interactions with gravitational 2D solitons in section 3. Then, in section 4, we construct exact solutions for Ricci flows depending 'anisotropically' on coordinate $p=z+t$ when the metrics are certain offdiagonal deformations from the a pp-wave background to configurations with nonlinear interactions between pp-wave components and 3D solitonic waves. Section 5 is devoted to solutions describing flows of pp-waves depending on the 5th coordinate when the torsion is related completely antisymmetric tensor fields in sting gravity. Finally, in section 6, we conclude and discuss the obtained results.

\section{Off-Diagonal Ricci Flows}

We consider a linear quadratic element

$$
d s^{2}=g_{\alpha \beta} d u^{\alpha} d u^{\beta}=g_{i}\left(x^{k}\right)\left(e^{i}\right)^{2}+g_{a}\left(x^{k}, p\right)\left(e^{a}\right)^{2}
$$

with the coefficients stated with respect to the co-frame

$$
e^{\alpha}=\left(e^{i}=d x^{i}, e^{a}=d p^{a}+N_{i}^{a}\left(x^{k}, p\right) d x^{i}\right)
$$

being dual to the frame (funfbein)

$$
e_{\alpha}=\left(e_{i}=\frac{\partial}{\partial x^{i}}-N_{i}^{a} \frac{\partial}{\partial p^{a}}, e_{a}=\frac{\partial}{\partial p^{a}}\right) .
$$

In our formulas, the Einstein's summation rule on indices is adopted, the indices of type $i, k, \ldots$ run values $1,2,3$; the indices of type $a, b, \ldots$ run values 4,5 and the local coordinates split in the form $u^{\alpha}=\left(x^{i}, p^{a}\right)$, where $p^{4}=p$ will be written in brief. The metric and funfbein coefficients are parametrized by functions of necessary smooth class,

$$
\begin{aligned}
g_{1} & = \pm 1, g_{2,3}=q_{2,3}\left(x^{2}, x^{3}\right) \underline{g}_{2,3}\left(x^{2}, x^{3}\right), g_{4,5}=q_{4,5}\left(x^{i}, p\right) \underline{g}_{4,5}\left(x^{i}\right), \\
N_{i}^{4} & =w_{i}\left(x^{i}, p\right), N_{i}^{5}=n_{i}\left(x^{i}, p\right) .
\end{aligned}
$$

We consider that the coefficients $\underline{g}_{\alpha}$ are given by a metric in a theory of gravity (it may be a solution of gravitational field equations or, for instance, a conformal transform of such a solution) and the coefficients $q_{\alpha}, w_{i}$ and $n_{i}$ 
are supposed to define deformations to other classes of solutions. For our purposes, we can state any (pseudo) Euclidean signature.

In this paper, we shall work both with the so-called 'canonical distinguished connection' $\Gamma_{\beta \gamma}^{\alpha}=\left(L^{i}{ }_{j k}, L_{b k}^{a}, C_{j c}^{i}, C_{b c}^{a}\right)$ and the Levi-Civita connection $\underline{\Gamma}_{\beta \gamma}^{\alpha}$, see details in Refs. [9, [10]. The coefficients of these connections can be computed with respect to the local bases (3) and (2), when

$$
\underline{\Gamma}_{\beta \gamma}^{\alpha}=\left(L_{j k}^{i}, L_{b k}^{a}-\frac{\partial N_{k}^{a}}{\partial p^{b}}, C_{j c}^{i}+\frac{1}{2} g^{i k} \Omega_{j k}^{a} g_{c a}, C_{b c}^{a}\right)
$$

for

$$
\begin{aligned}
\Omega_{i j}^{a} & =e_{i} N_{j}^{a}-e_{j} N_{i}^{a}, L_{j k}^{i}=\frac{1}{2} g^{i r}\left[e_{k}\left(g_{j r}\right)+e_{j}\left(g_{k r}\right)-e_{r}\left(g_{j k}\right)\right], \\
L_{b k}^{a} & =e_{b}\left(N_{k}^{a}\right)+\frac{1}{2} g^{a c}\left[e_{k}\left(g_{b c}\right)-g_{d c} e_{b}\left(N_{k}^{d}\right)-g_{d b} e_{c}\left(N_{k}^{d}\right)\right] \\
C_{j c}^{i} & =\frac{1}{2} g^{i k} e_{k}\left(g_{j k}\right), C_{b c}^{a}=\frac{1}{2} g^{a d}\left[e_{c}\left(g_{b d}\right)+e_{b}\left(g_{d c}\right)-e_{d}\left(g_{b c}\right)\right] .
\end{aligned}
$$

The connection $\Gamma_{\beta \gamma}^{\alpha}$ is compatible with the metric (11), i.e. it defines a covariant derivative $D_{\gamma}$ satisfying the conditions $D_{\gamma} g_{\alpha \beta}=0$. The torsion $T_{\beta \gamma}^{\alpha}=\Gamma_{\beta \gamma}^{\alpha}-\Gamma_{\gamma \beta}^{\alpha}$ vanishes partially, $T_{j k}^{i}=0$ and $T_{b c}^{a}=0$ but there are nontrivial components

$$
T_{i j}^{a}=\Omega_{i j}^{a}, T_{j a}^{i}=C_{j a}^{i}, T_{b j}^{a}=e_{b}\left(N_{j}^{a}\right)-L_{b j}^{a} .
$$

By definition, the Levi-Civita connection is torsionless and metric compatible, i.e. $\underline{T}_{\beta \gamma}^{\alpha}=0$ and $\underline{D}_{\gamma} g_{\alpha \beta}=0$. We note that in order to investigate flow solutions related to string gravity and spaces characterized by nonholonomic structures it is convenient to use connections with nontrivial torsion. The constraints resulting in zero torsion can be considered after certain general solutions have been constructed.

The normalized Ricci flows [4], with respect to the coordinate base $\partial_{\underline{\alpha}}=$ $\partial / \partial u^{\underline{\alpha}}$, are described by the equations

$$
\frac{\partial}{\partial \tau} g_{\underline{\alpha} \underline{\beta}}=-2 R_{\underline{\alpha} \underline{\beta}}+\frac{2 r}{5} g_{\underline{\alpha} \underline{\beta}},
$$

where the normalizing factor $r=\int R d V / d V$ is introduced in order to preserve the volume $V .{ }^{3}$ In this work, we consider that the parameter $\tau$ may define flows on any time like or extra dimension (5th) coordinate $x^{3}$, or $p$. We note

\footnotetext{
${ }^{3}$ we underlined the indices with respect to the coordinate bases in order to distinguish them from those defined with respect to the 'N-elongated' local bases (3) and (2)
} 
that we use the Ricci tensor $R_{\underline{\alpha} \underline{\beta}}$ and scalar curvature $R=g \underline{\underline{\alpha}} \underline{\beta} R_{\underline{\alpha} \underline{\beta}}$ computed for the connection $\Gamma$ but further will shall constrain the coefficients $N_{j}^{a}$ in such a manner that

$$
R_{\alpha \beta}=\underline{R}_{\alpha \beta}
$$

in order to define limits to subclasses of solutions related to the Einstein spaces. ${ }^{4}$

With respect to the funfbeins (3) and (2), when

$$
e_{\alpha}=e_{\alpha}^{\underline{\alpha}} \partial_{\underline{\alpha}} \text { and } e^{\alpha}=e_{\underline{\alpha}}^{\alpha} d u^{\underline{\alpha}}
$$

for the frame transforms respectively parametrized in the form

$$
e_{\alpha}^{\underline{\alpha}}=\left[\begin{array}{cc}
e_{i}^{\underline{i}}=\delta_{i}^{\underline{i}} & e_{i}^{\underline{a}}=N_{i}^{b} \delta_{b}^{\underline{a}} \\
e_{a}^{\underline{i}}=0 & e_{a}^{\underline{a}}=\delta \underline{a}
\end{array}\right] \text { and } e_{\underline{a}}^{\alpha}=\left[\begin{array}{cc}
e^{i}{ }_{\underline{\alpha}}=\delta_{\underline{i}}^{i} & e^{b}{ }_{\underline{i}}=-N_{k}^{b} \delta_{\underline{i}}^{k} \\
e_{\underline{a}}^{i^{i}}=0 & e_{\underline{a}}^{a}=\delta_{\underline{a}}^{a}
\end{array}\right] \text {, }
$$

where $\delta_{\underline{i}}^{i}$ is the Kronecher symbol, the Ricci flow equations (6) can be written

$$
\begin{aligned}
\frac{\partial}{\partial \tau} g_{i i} & =-2 R_{i i}+2 \lambda g_{i i}-g_{c d} \frac{\partial}{\partial \tau}\left(N_{i}^{c} N_{i}^{d}\right), \text { for } i=2,3 ; \\
\frac{\partial}{\partial \tau} g_{a a} & =-2 R_{a a}+2 \lambda g_{a a} ; \text { for } a=4,5 ; \\
R_{\alpha \beta} & =0 \text { for } \alpha \neq \beta,
\end{aligned}
$$

where $\lambda=r / 5$ and $g_{i j}$ and $g_{a b}$ are defined by the ansatz (11). The nontrivial components of the Ricci tensor $R_{\alpha \beta}$ are computed (see details of a similar calculus in Refs. 9, 10])

$$
\begin{aligned}
& R_{2}^{2}=R_{3}^{3}=\frac{1}{2 g_{2} g_{3}}\left[\frac{g_{2}^{\bullet} g_{3}^{\bullet}}{2 g_{2}}+\frac{\left(g_{3}^{\bullet}\right)^{2}}{2 g_{3}}-g_{3}^{\bullet \bullet}+\frac{g_{2}^{\prime} g_{3}^{\prime}}{2 g_{2}}+\frac{\left(g_{2}^{\prime}\right)^{2}}{2 g_{3}}-g_{2}^{\prime \prime}\right], \\
& R_{4}^{4}=R_{5}^{5}=\frac{1}{2 g_{4} g_{5}}\left[-g_{5}^{* *}+\frac{\left(g_{5}^{*}\right)^{2}}{2 g_{5}}+\frac{g_{4}^{*} g_{5}^{*}}{2 g_{4}}\right],
\end{aligned}
$$

where the functions $w_{i}$ and $n_{i}$ satisfy respectively the equations

$$
\begin{aligned}
-2 g_{5} R_{4 i} & =w_{i} \beta+\alpha_{i}=0 \\
-2 \frac{g_{4}}{g_{5}} R_{5 i} & =n_{i}^{* *}+\gamma n_{i}^{*}=0
\end{aligned}
$$

for

$$
\begin{aligned}
\alpha_{i} & =\partial_{i} g_{5}^{*}-g_{5}^{*} \partial_{i} \ln \sqrt{\left|g_{4} g_{5}\right|}, \beta=g_{5}^{* *}-g_{5}^{*}\left(\ln \sqrt{\left|g_{4} g_{5}\right|}\right)^{*}, \\
\gamma & =3 g_{5}^{*} / 2 g_{5}-g_{4}^{*} / g_{4}, \text { for } g_{4}^{*} \neq 0, g_{5}^{*} \neq 0,
\end{aligned}
$$

\footnotetext{
${ }^{4}$ the tensor $\underline{R}_{\underline{\alpha} \underline{\beta}}$ being computed for the connection $\underline{\Gamma}$
} 
defined by $g_{4}$ and $g_{5}$ as solutions of equations (92). In the above presented formulas, it was convenient to write the partial derivatives in the form $a^{\bullet}=$ $\partial a / \partial x^{2}, a^{\prime}=\partial a / \partial x^{3}$ and $a^{*}=\partial a / \partial p$.

In Refs. [9, 10] (see, for instance, the formulas (160) and (161) in the Appendix to Ref. [10]), we proved that for an ansatz of type (10) stated by data (41) the conditions (7), generating Einstein spaces, transform into certain systems of partial differential equations for the off-diagonal metric coefficients

$$
\begin{aligned}
2 g_{5} w_{k}^{* *}+w_{k}^{*} g_{5}^{*} & =0 \\
n_{k}^{*} g_{5}^{*} & =0
\end{aligned}
$$

and

$$
\begin{aligned}
w_{2}^{\prime}-w_{3}^{\bullet}+w_{3} w_{2}^{*}-w_{2} w_{3}^{*}=0 \\
n_{2}^{\prime}-n_{3}^{\bullet}+w_{3} n_{2}^{*}-w_{2} n_{3}^{*}=0,
\end{aligned}
$$

Such constraints are necessary also in order to generate Ricci flows of the Einstein metrics.

In the next sections, we shall construct explicit examples of exact solutions, of equations (8)-(13) for metric anzats of type (11) defined by data (41), describing flows of 4D metrics into 5D spacetimes.

\section{$3 \quad$ Ricci flows on coordinate $\tau=v$}

Let us consider a 5D metric

$$
d s^{2}=-d x^{2}-d y^{2}+\frac{1}{8 f(x, y, p)} d v^{2}-2 f(x, y, p) \delta p^{2} \pm\left(d p^{5}\right)^{2},
$$

where

$$
\delta p=d p-\frac{1}{4 f(x, y, p)} d v .
$$

For $p=z+t$ and $v=t-z$, the ansatz (17) defines trivial extensions on the 5th coordinate $p^{5}$ of the $4 \mathrm{D}$ pp-wave solutions of the vacuum Einstein equations if the function $f(x, y, p)$ satisfies the condition

$$
f_{x x}+f_{y y}=0,
$$

were $f_{x}=\partial f / \partial x$, see the original paper [1] and recent developments in string theory [2] ${ }^{5}$ Usually, one considers pp-waves parametrized in the form

$$
f=f_{1}(x, y) f_{2}(p),
$$

\footnotetext{
${ }^{5}$ one can be performed both space or time like 5 th dimension extensions
} 
when, for instance, $f_{1}(x, y)=x^{2}-y^{2}$ and $f_{2}(p)=\sin p$, or $f_{1}(x, y)=$ $x y\left(x^{2}+y^{2}\right)$ and $f_{2}(p)=\exp \left[b^{2}-p^{2}\right]^{-2}$ when $|p|<b$ and $f=0$ for $|p| \geq b$.

The pp-wave metric (17) is a particular case of off-diagonal ansatz (11) when, for instance, the data (44) are stated by nonzero coefficients

$$
q_{\alpha}=1, \underline{g}_{1,2}=-1, \underline{g}_{3}=-1 / 8 f, \underline{g}_{4}=-2 f, \underline{g}_{5}= \pm 1, \underline{w}_{3}=-\frac{1}{4 f}
$$

for the local coordinates parametrized in the form $x^{i}=(x, y, v)$ and $p^{a}=$ $\left(p, p^{5}\right)$. In this section, we shall deform the metric (17) by introducing some values $q_{\alpha}\left(x^{i}, p\right)$ and $N_{i}^{a}\left(x^{i}, p\right)$, see (4), generating new solutions of the Ricci flow equations (8), (9) and (12), (13). We shall search solutions for 'deformations' to an ansatz of type (17) stated in the form

$$
\begin{aligned}
d s^{2} & =-d x^{2}-g_{2}(v)\left[d y^{2}-d v^{2}\right]-g_{4}(x, y, p) \delta p^{2} \pm g_{5}(p)\left(\delta p^{5}\right)^{2} \\
\delta p & =d p+w_{2}(x, y) d y+w_{3}(p) d v \\
\delta p^{5} & =d p^{5}+n_{i}(x, y, v, p) d x^{i}
\end{aligned}
$$

The coefficients from (20) are defined by multiplying the data (19) on respective 'polarization' functions when

$$
\begin{aligned}
g_{2}(v) & =\eta_{2}(y, v) \underline{g}_{2}, g_{3}(v)=\eta_{3}(x, y, v, p) \underline{g}_{3}=g_{2}(v), \\
g_{4}(x, y, p) & =\eta_{4}(x, y, v, p) \underline{g}_{4}, g_{5}(p)=\eta_{5}(p) \underline{g}_{5}, w_{3}(p)=\eta_{3}^{[w]}(x, y, v, p) \underline{w}_{3},
\end{aligned}
$$

and by considering certain additional/ modified 'N-coefficients' $w_{2}(x, y)$ and $n_{i}(x, y, p)$.

Computing the components $R_{i j}$ of the Ricci tensor (10) for the connection $\Gamma_{\beta \gamma}^{\alpha}$ defined by ansatz (20), we write the Ricci flow equation (18) in the form

$$
\theta^{\prime \prime}+\frac{1}{\theta}\left(\theta^{\prime}\right)^{2}-\theta \theta^{\prime}+2 \lambda \theta=0
$$

where $\theta(v)=g_{2}(v)=g_{3}(v)$. This equation is investigated in Ref. [12]. Stating $\mu(\theta)=d \theta(v) / d v$, we obtain the Abel equation

$$
\mu \frac{d \mu}{d \theta}+\frac{1}{\theta} \mu^{2}-\theta \mu+2 \lambda \theta=0 .
$$

A similar equation, without the term $+\frac{1}{\theta} \mu^{2}$,

$$
\mu \frac{d \mu}{d \theta}-\theta \mu+2 \lambda \theta=0
$$

was considered for various classes of exact off-diagonal solutions in gravity, see discussions and references in Refs. [9, 10. For $\lambda \rightarrow 0$, we get a Bernoulli 
type equation. We conclude that for the ansatz (20) the Ricci flow of components $g_{2}(v)=g_{3}(v)$ are governed by explicit solutions of the Abel or Bernoulli equations.

For a subclass of solutions when $g_{4,5}$ and $N_{i}^{a}$ in (20) do not depend on variable $x^{3}=v$, the terms $\frac{\partial}{\partial \tau} g_{a a}$ in (9) vanish, i.e. $R_{4}^{4}=R_{5}^{5}=\lambda$,

$$
g_{5}^{*}\left[-\frac{g_{5}^{* *}}{g_{5}^{*}}+\frac{g_{5}^{*}}{2 g_{5}}+\frac{g_{4}^{*}}{2 g_{4}}\right]=2 g_{4} g_{5} \lambda .
$$

The general procedure of constructing exact solutions of the systems of equations (23), (12) and (13) is described in Appendices to Refs. [9, 10]. Here we present the final results of such computations describing $4 \mathrm{D}$ generic offdiagonal Einstein metrics (by straightforward computations one can be verified that the given sets of functions really solve the field equations): One has

where

$$
g_{4}=\zeta \eta_{4}^{[\lambda=0]} \underline{g}_{4} \text { and } g_{5}=\eta_{5}(p) \underline{g}_{5}
$$

$$
\begin{aligned}
\zeta(x, y, p) & =1+\frac{\lambda}{8} h_{0}^{2}(x, y) \int \sqrt{\left|\eta_{5} \eta_{5}^{*}\right|} d p \\
\eta_{4}^{[\lambda=0]}(x, y, p) & =\frac{h_{0}^{2}(x, y)}{f_{1}(x, y)}\left[f_{2}^{*}-\frac{f_{2} f_{2}^{* *}}{2\left(f_{2}^{*}\right)^{2}}\right], \eta_{5}(p)=\left(f_{2}\right)^{4} /\left(f_{2}^{*}\right)^{2}
\end{aligned}
$$

for an arbitrary function $h_{0}(x, y)$, a given $\underline{g}_{4}=-2 f_{1}(x, y) f_{2}(p)$ defined by the 'non-deformed' pp-wave solution, and for $\underline{g}_{5}= \pm 1$, depending on the signature of the 5 th dimension. We can satisfy the conditions (15) and (16) by any functions

$$
w_{2}=w_{2}(x, y) \text { and } w_{3}=\left[f_{2}(p)\right]^{-1}
$$

and any

$$
n_{2}=n_{2}(x, y, v) \text { and } n_{3}=n_{3}(x, y, v)
$$

for which $n_{2}^{\prime}-n_{3}^{\bullet}=0$ and $n_{2,3}^{*}=0$.

In explicit form, such Ricci flow solutions are described by the generic off-diagonal metric

$$
\begin{aligned}
d s^{2}= & -d x^{2}-\theta(v)\left[d y^{2}-d v^{2}\right]-2 h_{0}^{2}(x, y) f_{2}(p)\left[f_{2}^{*}-\frac{f_{2} f_{2}^{* *}}{2\left(f_{2}^{*}\right)^{2}}\right] \times \\
& \left(1+\frac{\lambda}{4} h_{0}^{2}(x, y) \int\left(f_{2}\right)^{3} \sqrt{\left|\frac{f_{2}}{f_{2}^{*}}-\frac{\left(f_{2}\right)^{2}}{2\left(f_{2}^{*}\right)^{3}}\right|} d p\right) \delta p^{2} \\
& \pm\left(f_{2}\right)^{4} /\left(f_{2}^{*}\right)^{2}\left(\delta p^{5}\right)^{2} \\
\delta p= & d p+w_{2}(x, y) d y+\left[f_{2}(p)\right]^{-1} d v \\
\delta p^{5}= & d p^{5}+n_{2}(x, y, v) d y+n_{3}(x, y, v) d v .
\end{aligned}
$$


By straightforward computations, we can verify that the coefficients $g_{2}(v)$ $=g_{3}(v)$, solving (22), and the coefficients (24)-(27) for the ansatz (20) define a class of exact solutions of equations (8) and (9) describing off-diagonal Ricci flows of 4D Einstein metrics into certain 5D spacetimes. The solutions depend on arbitrary integration functions $h_{0}(x, y), w_{2}(x, y)$ and $n_{2,3}(x, y, v)$, for which $n_{2}^{\prime}=n_{3}^{\bullet}$. We emphasize that such functions can not be completely eliminated by coordinate transforms: they describe certain classes of spacetimes which can be further constrained to satisfy explicit symmetry conditions for nonlinear gravitational interactions and flows. For instance, one can be considered the case when $h_{0}(x, y)$ is a solution of a $2 \mathrm{D}$ solitonic equation, for instance, of the sine-Gordon equation ( $\mathrm{SG}$ ),

$$
\pm \frac{\partial^{2} h_{0}}{\partial x^{2}} \mp \frac{\partial^{2} h_{0}}{\partial y^{2}}=\sin \left(h_{0}\right) \text {. }
$$

This way we generate a class of solutions describing nonlinear off-diagonal interactions of gravitational pp-waves and 2D solitonic waves with nontrivial Ricci flows of some components of metric satisfying the corresponding Bernoulli, or Abel, type equation. If $g_{2}^{\prime}\left(v_{0}\right)=g_{3}^{\prime}\left(v_{0}\right)=0$ in a point $v=v_{0}$, the flow solutions are approximated by a set of exact off-diagonal solutions of the vacuum Einstein equations with nontrivial cosmological constant defining solitonic pp-waves. This class of metrics is generated by corresponding off-diagonal deformations of the $4 \mathrm{D} \mathrm{pp-wave} \mathrm{metric} \mathrm{embedded} \mathrm{into} 5 \mathrm{D}$ spacetime, see ansatz (17).

We can generalize our solutions by introducing certain dependencies on the "flow" coordinate $v$ in $w_{2,3}(x, y, v)$ and $n_{2,3}(x, y, v)$ not subjected to the conditions (15) and (16), for instance, by supposing that some such components are solutions of the 3D solitonic equations. This way, one generates Ricci flows of pp-waves nonlinearly interacting with 3D solitons inducing nontrivial torsion (5) which under corresponding conditions can be related to a nontrivial torsion field in string gravity, Einstein-Cartan gravity, or certain models of metric-affine, Finsler and/or noncommutative generalizations of gravity, see Refs. [9, 10] and the section [5] in this work.

\section{Ricci flows on coordinate $\tau=p$}

We analyze an ansatz

$$
\begin{aligned}
d s^{2} & =-d x^{2}+g_{2}\left(x^{i}\right) d y^{2}+g_{3}\left(x^{i}\right) d v^{2}+g_{4}\left(x^{i}, p\right)(\delta p)^{2}+g_{5}\left(x^{i}, p\right)\left(\delta p^{5}\right)^{2} \\
\delta p & =d p+w_{3}\left(x^{i}, p\right) d v, \delta p^{5}=d p^{5}+n_{3}\left(x^{i}, p\right) d v
\end{aligned}
$$


for $g_{4,5}=\eta_{4,5}(x, y, v, p) \underline{g}_{4,5}$, with $\underline{g}_{4}=-2 f_{1}(x, y) f_{2}(p)$ and $\underline{g}_{4}= \pm 1$, defined by a solution of $\mathrm{pp}$-wave equation (18), when the local coordinates are stated in the form $x^{i}=(x, y, v)$ and $p^{a}=\left(p, p^{5}\right)$. We search for solutions of the system of equations (8), (9) and (12), (13) beginning with solutions of $R_{a}^{a}=0$, see (11), of type

$$
\sqrt{\left|g_{4}\right|}=h_{0}\left(\sqrt{\left|g_{5}\right|}\right)^{*}, h_{0}=\text { const }
$$

when the term $g_{c d} \frac{\partial}{\partial p}\left(N_{i}^{c} N_{i}^{d}\right)$ in (8) is constrained to be zero as the solution of the equation

$$
g_{4} \frac{\partial}{\partial p}\left(w_{3}\right)^{2}+g_{5} \frac{\partial}{\partial p}\left(n_{3}\right)^{2}=0
$$

for $N_{i}^{4}=w_{i}$ and $N_{i}^{5}=n_{i}$ where $\frac{\partial}{\partial p} w_{3}=w_{3}^{*}(x, y, v, p)$. If the relation (29) holds, one has $\alpha_{i}=\beta=0$, see formulas (14). This allows us to consider any values $w_{i}$ in (12). For instance, we can take $w_{3}(x, y, v, p) \neq 0$ and $w_{1}=w_{2}=0{ }^{6}$. For the ansatz (28) $)$, parametrizing $\eta_{a}=\eta_{a}^{[1]}(x, y, v) \eta_{a}^{[2]}(p)$, one obtains from (29) that

$$
\eta_{5}^{[1]}=\left|2 f_{1} \eta_{4}^{[1]}\right|
$$

and

$$
\sqrt{\left|2 f_{2}(p) \eta_{4}^{[2]}(p)\right|}=h_{0}\left(\sqrt{\left|\eta_{5}^{[2]}(p)\right|}\right)^{*} .
$$

This is compatible with the equation (9) if $\sqrt{|2 \lambda|} h_{0}=1$, when

$$
\eta_{5}^{[2]}(p)=\eta_{5[0]} e^{2 \lambda p}=f_{2}(p) \eta_{4}^{[2]}(p)
$$

for $\eta_{5[0]}=$ const.

A nontrivial solution of (13) and (29) can be written in the form

$$
n_{1,2}=0, n_{3}=n_{3[1]}(x, y, v)+n_{3[2]}(x, y, v) \int \frac{e^{-\lambda p} d p}{\sqrt{\left|f_{2}(p)\right|}}
$$

where $n_{3[1,2]}(x, y, v)$ are some integration functions. We can compute $w_{3}$ by integrating (30) for defined $g_{4,5}$ and $n_{3}$,

$$
w_{3}=\left|2 f_{1}(x, y)\left[f_{2}(p)-\int f_{2}(p) d p\right]\right|^{1 / 2} n_{3}(x, y, v, p) .
$$

\footnotetext{
${ }^{6}$ for simplicity, we chose a 'minimal' extension of the set of such functions
} 
The final step in constructing this class of solutions is to solve the equation (8) which for the ansatz (28), see formulas (111), and the condition (30) transforms into

$$
\frac{g_{2}^{\bullet} g_{3}^{\bullet}}{2 g_{2}}+\frac{\left(g_{3}^{\bullet}\right)^{2}}{2 g_{3}}-g_{3}^{\bullet \bullet}+\frac{g_{2}^{\prime} g_{3}^{\prime}}{2 g_{2}}+\frac{\left(g_{2}^{\prime}\right)^{2}}{2 g_{3}}-g_{2}^{\prime \prime}=2 g_{2} g_{3} \lambda
$$

The general solution of this equation can be written in the form

$$
\varpi=g_{[0]} \exp \left[a_{2} \widetilde{x}^{2}(y, v)+a_{3} \widetilde{x}^{3}(y, v)\right],
$$

where $g_{[0]}$ and $a_{2,3}$ are some constants and the functions $\widetilde{x}^{2,3}(y, v)$ define a coordinate transform $x^{2,3} \rightarrow \widetilde{x}^{2,3}$ for which the 2D line element becomes conformally flat,

$$
g_{2}\left(x^{i}\right) d y^{2}+g_{3}\left(x^{i}\right) d v^{2} \rightarrow \varpi(y, v)\left[\left(d \widetilde{x}^{2}\right)^{2}+\left(d \widetilde{x}^{3}\right)^{2}\right]
$$

with $\psi=\ln |\varpi|$ satisfying the equation

$$
\psi^{\bullet \bullet}-\psi^{\prime \prime}=-2 \lambda .
$$

Summarizing the coefficients for the ansatz (28), we get the metric

$$
\begin{aligned}
d s^{2}= & -d x^{2}-e^{\psi(y, v)}\left[\left(d \widetilde{x}^{2}(x, y)\right)^{2}-\left(d \widetilde{x}^{3}(x, y)\right)^{2}\right] \\
& -2 f(x, y, p) \eta_{4}^{[1]}(x, y, v) \eta_{4}^{[2]}(p)(\delta p)^{2} \pm \eta_{5}^{[0]} e^{2 \lambda p}\left(\delta p^{5}\right)^{2}, \\
\delta p= & d p+w_{3}(x, y, v, p) d v, \delta p^{5}=d p^{5}+n_{3}(x, y, v, p) d v,
\end{aligned}
$$

depending on 'polarization' (integration) functions $\eta_{4}^{[1]}$ and $n_{3[1,2]}$ and constant $\eta_{5}^{[0]}$ with nontrivial $\mathrm{N}$-coefficients $n_{3}$ and $w_{3}$ defined respectively by the equations (31) and (321) and $\psi(y, v)$ being a solution of (33). We can state in explicit form a nonlinear superposition of a pp-wave $f=f_{1} f_{2}$ and a 3D soliton when $\eta_{4}^{[1]}(x, y, v)$ is a solution, for instance, of the $(2+1)$-dimensional SG equation,

$$
-\left(\eta_{4}^{[1]}\right)^{\bullet \bullet}+\left(\eta_{4}^{[1]}\right)^{\prime}+\frac{\partial^{2}}{\left(\partial x^{1}\right)^{2}}\left(\eta_{4}^{[1]}\right)=\sin \left(\eta_{4}^{[1]}\right) .
$$

It should be noted that the metric (34) describes solitonic pp-wave flows for the Ricci tensor defined for the canonical distinguished connection $\Gamma_{\beta \gamma}^{\alpha}$ possessing certain nontrivial torsion coefficients. We can restrict the integral varieties (of solutions) by considering only some nontrivial coefficients $w_{3}(v)$ and $n_{3}(v)$ depending on variable $x^{3}=v$ with the integration functions subjected to the conditions (15) and (16). In such cases, we extract exact solutions for flows (on coordinate $p=z+t$ ) of a 4D Einstein space propagating as a nontrivial embedding into $5 \mathrm{D}$ spacetimes. 


\section{Flows on Extra Dimension Coordinate}

Other classes of exact solutions for Ricci flows can be constructed by deforming the metric (17) as in previous section but with reparametrized coordinates $x^{i}=(x, y, v)$ and $p^{a}=\left(p^{5}, p\right)$, by multiplying the coefficients $\underline{g}_{\alpha}$ on polarization functions

$$
\begin{aligned}
& \eta_{1}=1, \eta_{2}=\eta_{2}(y, v), \eta_{3}=f_{2}(p) \eta_{3}^{[1]}(y, v), \\
& \eta_{4}=\left[f_{2}(p)\right]^{-1} \eta_{4}^{[1]}(x, y, v) \eta_{4}^{[2]}\left(p^{5}\right), \eta_{5}=\eta_{5}\left(x, y, v, p^{5}\right)
\end{aligned}
$$

and then following similar geometric constructions when the dependence on coordinate $p^{5}$ for a pp-wave ${ }^{e x t} f\left(x, y, p^{5}\right)$ is emphasized instead of a usual function $f(x, y, p)$ for $4 \mathrm{D}$ solutions. The derived formulas and equations are similar but with $p \longleftrightarrow p^{5}$ defining flows on the 5 th coordinate $p^{5}$. Such solutions are parametrized by the ansatz

$$
\begin{aligned}
d s^{2}= & -d x^{2}-e^{\psi(y, v)}\left[\left(d \widetilde{x}^{2}(x, y)\right)^{2}-\left(d \widetilde{x}^{3}(x, y)\right)^{2}\right] \\
& -2 f\left(x, y, p^{5}\right) \eta_{4}^{[1]}(x, y, v) \eta_{4}^{[2]}\left(p^{5}\right)\left(\delta p^{5}\right)^{2} \pm \eta_{5}^{[0]} e^{2 \lambda p^{5}}(\delta p)^{2} \\
\delta p^{5}= & d p^{5}+w_{3}\left(x, y, v, p^{5}\right) d v, \delta p=d p+n_{3}\left(x, y, v, p^{5}\right) d v
\end{aligned}
$$

with the coefficients defined by equations of type (31), (32) and (33), and can be constrained, for certain $w_{3}(v)$ and $n_{3}(v)$ to define flows of the Einstein spaces for the Levi-Civita connection $\underline{\Gamma}$.

For solutions with nontrivial torsion (5), we can relate the Ricci tensor flows to a source defined by the strength (torsion)

$$
H_{\mu \nu \rho}=e_{\mu} B_{\nu \rho}+e_{\rho} B_{\mu \nu}+e_{\nu} B_{\rho \mu}
$$

of an antisymmetric field $B_{\nu \rho}$, when

$$
R_{\mu \nu}=-\frac{1}{4} H_{\mu}^{\nu \rho} H_{\nu \lambda \rho}
$$

and

$$
D_{\lambda} H^{\lambda \mu \nu}=0,
$$

see details on string gravity, for instance, in Refs. [13. Here, we also note that the 3D string gravity is used for a modified Ricci flow analysis of the Thurston geometrization conjecture [14] but our approach is oriented to constructing exact solutions. The conditions (36) and (37) are satisfied by the ansatz

$$
H_{\mu \nu \rho}=\widehat{Z}_{\mu \nu \rho}+\widehat{H}_{\mu \nu \rho}=\lambda_{[H]} \sqrt{\left|g_{\alpha \beta}\right|} \varepsilon_{\nu \lambda \rho}
$$


where $\varepsilon_{\nu \lambda \rho}$ is completely antisymmetric and the distorsion (from the LeviCivita connection) $\widehat{Z}_{\mu \nu \rho}$ is defined by the torsion tensor (15). ${ }^{7}$

For nontrivial $\mathrm{H}$-fields of type (38), we have to re-define the 'cosmological' constant, $\lambda \rightarrow \lambda+\lambda_{[H]}$, respectively in the formulas (31)-(33) and (35) and write, for instance, $e^{2\left(\lambda+\lambda_{[H]}\right) p^{5}}$ instead of $e^{2 \lambda p^{5}}$.

\section{Conclusions}

We constructed three classes of exact solutions of Ricci flow equations in 5D spacetimes. Despite the presence in literature of rather sophisticate mathematical considerations [4] on existence of solutions no explicit constructions attempting such constructions were performed excepting the linearized approach [15], which also allows to generate exact solutions but for lower dimensions. The Ricci flows are described by nonlinear systems of second order parabolic differential equations which can not approached by the usual techniques of constructing exact solutions with diagonalizable metrics in gravity theories. The 'anholonomic frame method' [8, 9, 10] is a general geometric one allowing to construct generic off-diagonal exact solutions which naturally can be related both to solutions of the Einstein equations and Ricci flows.

In this paper, we analyzed flows of pp-waves on nontrivial solitonic backgrounds of 5D spacetimes. Such equations (at least for normalized flows and certain ansatz for string gravity) contain terms with nontrivial cosmological constants. There is some similarity with the solutions defining Kaigorodov spaces [1], constructed as homogeneous Einstein spaces induced by ppwaves propagating in anti-de Sitter space (it is conjectured in modern literature [2, 14] that for a string/M-theory on the Kaigorodov spaces a compact manifold is dual to a conformal field theory in an infinitely-boosted frame with constant momentum density). ${ }^{8}$

The off-diagonal metric coefficients describing flows of superpositions of pp-waves and solitons have certain analogy with previous results on pp-waves in higher dimensions and non-relativistic versions of Kaluza-Klein monopole

\footnotetext{
${ }^{7}$ We emphasize that our $\mathrm{H}$-field ansatz is different from those already used in string gravity when $\widehat{H}_{\mu \nu \rho}=\lambda_{[H]} \sqrt{\left|g_{\alpha \beta}\right|} \varepsilon_{\nu \lambda \rho}$ [13]. In our approach, we define $H_{\mu \nu \rho}$ and $\widehat{Z}_{\mu \nu \rho}$ from respective ansatzes for the $\mathrm{H}$-field and nonholonomically deformed metric, compute the torsion tensor for the canonical distinguished connection $\Gamma$ and after that it is possible to find the 'deformed' H-field, $\widehat{H}_{\mu \nu \rho}=\lambda_{[H]} \sqrt{\left|g_{\alpha \beta}\right|} \varepsilon_{\nu \lambda \rho}-\widehat{Z}_{\mu \nu \rho}$.

${ }^{8}$ One should be noted that nonholonomic deformations of charged Kaigorodov spaces can be generated from the data $\underline{g}_{\alpha}$ in (4) restricted to define a particular case of exact solutions (36) and (37) defining Einstein-Maxwell configurations with nontrivial cosmological constants as in Ref. [16].
} 
17. locally anisotropic Taub-NUT, soliton-spinor waves, wormholes and solitonically moving black holes in higher dimensions [18, 8]. Here, we emphasize that the approach elaborated in this work contains general off-diagonal terms (so called $\mathrm{N}$-coefficients) not restricted to the Kaluza-Klein conditions (related to linearizations and compactifications on extra-dimension coordinates). We can consider both vacuum gravitational pp-wave effects and Ricci flows, for instance, self-consistently imbedded into nontrivial solitonic backgrounds and spinor waves and/or superpositions with black hole metrics.

Our Ricci flow solutions describe nonlinear deformations and gravitational interactions with nontrivial pp-waves and solitonic backgrounds. In a particular case (for the ansatz for Kaigorodov solutions) they transform into homogeneous configurations with anti-de Sitter constant, but, in general, they are related to nonhomogeneous Einstein spaces and their string generalizations. Such metrics are generic off-diagonal, depend on classes of functions on one, two, three and four variables and can be with generalized (non-Killing and non-group) symmetries.

The solitonic flows of $\mathrm{pp}$-waves can be considered both on time like and/or extra dimension coordinates, in 4D and/or 5D gravity.

\section{Acknowledgments}

The author is grateful for discussions and substantial support to T. Wolf, S. Anco and M. Visinescu. He also thanks for support of Fields Institute.

\section{References}

[1] A. Peres, Phys. Rev. Lett. 3, 571 (1959).

[2] D. Bernstein, J. Maldacena and H. Nastase, JHEP 0204, 013 (2002); R. Metsaev and A. Tseytlin, Phys. Rev. D65, 126004 (2002); M. Cvetic, H. Lu and C. Pope, Nucl. Phys. B644, 65 (2002); A. Coley, R. Milso, N. Pelavas, V. Pravda, A. Pravdova and R. Zalaletdinov, Phys. Rev. D67, 104020 (2003).

[3] V. A. Belinski and V. E. Zakharov, Sov. Phys.-JETP 48, 985 (1978) [translated from Zh. Exp. Teor. Fiz. 75, 1953 (1978)]; V. Belinski and E. Verdaguer, Gravitational Solitons (Cambridge University Press, 2001); S. Vacaru and D. Singleton, Class. Quant. Gravity 19, 2793 (2002).

[4] R. S. Hamilton, J. Differential Geom. 17, 255 (1982); R. S. Hamilton, Contemporary Mathematics 71, 237 (1988); G. Perelman, The 
Entropy Formula for the Ricci Flow and its Geometric Applications, math.DG/0211159; H.-D. Cao, B. Chow, S.-C.Chu and S.-T.Yau (eds.), Collected Papers on Ricci Flow (International Press, Somerville, 2003); I. Bakas, JHEP 0308, 013 (2003).

[5] D. Kramer, H. Stephani, E. Herdlt and M. A. H. MacCallum, Exact Solutions of Einstein's Field Equations (Cambridge University Press, 1980); 2d edition (2003).

[6] J. Bicak, in: Lect. Notes. Phys. 540 (2000), p. 1-126

[7] R. Geroch, J. Math. Phys. 13, 233 (1972).

[8] S. Vacaru, JHEP 09, 011 (1998); S. Vacaru, J. Math. Phys. 46, 042503 (2005).

[9] S. Vacaru, E. Gaburov and D. Gontsa, A Method of Constructing OffDiagonal Solutions in Metric-Affine and String Gravity, hep-th/0310133, in: Clifford and Riemann- Finsler Structures in Geometric Mechanics and Gravity, Selected Works, by S. Vacaru, P. Stavrinos, E. Gaburov and D. Gonţa. Differential Geometry - Dinamical Systems, Monograph 7 (Geometry Balkan Press, 2006), p. 10- 162; www.mathem.pub.ro/dgds/mono/va-t.pdf and gr-qc/0508023

[10] S. Vacaru, Nonholonomic Deformations of Disk Solutions and Algebroid Symmetries in Einstein and Extra Dimension Gravity, gr-qc/0504095.

[11] V. R. Kaigorodov, Dokl. Akad. Nauk SSSR 146, 793 (1962); English translation in: Sov. Phys. Doklady 7, 893 (1963).

[12] E. Kamke, Differntialgleichungen, Losungsmethoden und Lonsungen: I. Gewohnliche Differentialgleichungen (Leipzig, 1959).

[13] P. Deligne, P. Etingof, D. S. Freed et all (eds.), Quanum Fields and Strings: A Course for Mathematicians, Vols 1 and 2, Institute for Adavanced Study (American Mathematical Society, 1994); J. Polchinski, String Theory, Vols 1 and 2 (Cambrdge University Press, 1998).

[14] J. Gegenberg and G. Kunstatter, Class. Quant. Grav. 21, 1197 (2004).

[15] S. A. Carstea and M. Visinescu, Mod. Phys. Lett. A 20, 2993 (2005).

[16] R.-G. Cai, Phys. Lett. B 572, 75 (2003). 
[17] C. Duval, P. Horvathy and G. W. Gibbons, Phys. Rev. D43, 3907 (1991).

[18] S. Vacaru and O. Tintareanu-Mircea, Nucl. Phys. B626, 239 (2002); S. Vacaru and F. C. Popa, Class. Quant. Gravity 18, 4921 (2001); S. Vacaru and D. Singleton, Class. Quant. Gravity 19, 2793 (2001). 